\title{
Diagnóstico tardío de la poliquistosis renal autosómica dominante en una población peruana
}

\section{Late diagnosis of autosomal dominant polycystic kidney disease in Peruvian population}

Correspondencia Percy Herrera-Añazco silamud@gmail.com

Recibido: 20/02/2017 Aprobado: 01/03/2017

Citar como: Herrera-Añazco P, Bazán-Lopez J, PachecoMendoza J, ValenzuelaRodriguez G. Diagnóstico tardio de la poliquistosis renal autosómica dominante en una población pervana. Acta Med Peru. 2017;34(1):72-3

\section{Percy Herrera-Añazco ${ }^{1,2}$, Jose L Bazán-Lopez³ , Josmel Pacheco-Mendoza4,} Germán Valenzuela-Rodriguez ${ }^{5}$

Hospital Nacional Dos de Mayo. Lima, Perú.

2 Universidad Peruana de Ciencias Aplicadas. Lima, Perú.

3 Universidad Peruana Cayetano Heredia. Lima, Perú.

4 Universidad San Ignacio de Loyola. Lima, Perú.

5 Centro de Investigación, Clínica Delgado, AUNA. Lima, Perú.

\section{Sr. Editor:}

La poliquistosis renal autosómico dominante (PQRAD) afecta alrededor de 12,5 millones de personas alrededor del mundo y, con el 6 a 10\% de los casos, es la cuarta etiología más frecuente entre pacientes con enfermedad renal crónica (ERC) que requieren hemodiálisis (HD) ${ }^{[1-3]}$. El surgimiento de alternativas terapéuticas como el Tolvaptan ha abierto la posibilidad de enlentecer la progresión de la enfermedad, por lo que es necesario identificar tempranamente a los pacientes que se beneficiarán de ésta terapia ${ }^{[1-3]}$. Además, permitirá instaurar otras medidas terapéuticas o dar consejería genética para el beneficio tanto de los pacientes como de su familia ${ }^{[1-3]}$. En el Perú, no existen datos epidemiológicos sobre PQRAD por lo que no es posible identificar aspectos perfectibles en el manejo de ésta enfermedad.

Se realizó un estudio observacional y descriptivo para identificar a los pacientes con ERC incidentes en HD que tuvieron como etiología a la PQRAD. Se incluyeron el en estudio aquellas personas con ERC incidentes en HD admitidas entre enero del 2012 y octubre del 2016 en el Hospital Nacional Dos de Mayo, un hospital de referencia nacional en Lima - Perú. Tantos los datos epidemiológicos como sociodemográficos fueron recolectados del registro epidemiológico del servicio de nefrología de dicho hospital. El diagnóstico de PQRAD fue hecho de acuerdo a criterios ultrasonográficos y antecedentes familiares ${ }^{[-3-3]}$.

De 553 pacientes evaluados, 15 (2,7\%) tenían el diagnóstico de PQRAD. La edad promedio fue de 50,33 años y 10 fueron hombres. Ocho pacientes fueron diagnosticados de ERC en el último mes antes de su ingreso a HD, cuatro entre en el segundo y sexto mes y tres luego del sexto mes. La tasa de filtración glomerular promedio con la que iniciaron $\mathrm{HD}$ fue de $4,03 \mathrm{~mL} / \mathrm{min}$ y sólo el $20,0 \%$ fue evaluado previamente por un nefrólogo.

Nuestros resultados muestran una baja frecuencia de PQRAD, siendo predominantemente de sexo masculino; similar a lo encontrado en países vecinos como Brasil ${ }^{[4]}$. La edad de ingreso a HD fue similar a otros estudios, los mismos que no encontraron diferencias según sexo; no obstante, el género masculino es considerado un determinante de peor pronóstico, lo que los lleva a necesitar HD de manera más temprana ${ }^{[4]}$. 
A pesar de que la PORAD es una enfermedad hereditaria y que han mejorado tanto el entendimiento epidemiológico de la enfermedad como los criterios diagnósticos y la consejería genética, nuestros pacientes tienen un diagnóstico tardío y pocos fueron vistos previamente por un nefrólogo. El diagnóstico tardío de la ERC en el Perú no es exclusivo de la PQRAD [5]; sin embargo, el ser una enfermedad hereditaria, es decir que el paciente tiene dentro de una familia otros miembros con la misma enfermedad, hace alarmante éste hecho. El Ministerio de Salud debería diseñar políticas de detección y manejo tempranos que incluyan equipos multidisciplinarios de especialistas calificados conocedores de la enfermedad, que identifiquen tempranamente a los pacientes y los cuales se beneficien de las terapias actuales de enlentecimiento de progresión de la enfermedad. Así mismo, se recomienda poner más interés en la planificación familiar de los pacientes portadores, intervención que incluye la consejería genética, el diagnóstico genético preimplantación y el acceso a fertilización in vitro, entre otros ${ }^{[1-3]}$.

\section{REFERENCIAS BIBLIOGRÁFICAS}

1. Chapman AB, Devuyst O, Eckardt KU, Gansevoort RT, Harris $\mathrm{T}$, Horie $\mathrm{S}$, et al. Autosomal-dominant polycystic kidney disease(ADPKD): executive summary from a Kidney Disease: Improving Global Outcomes (KDIGO) Controversies Conference. Kidney Int. 2015;88(1):17-27.

2. Ars E, Bernis C, Fraga G, Martínez V, Martins J, Ortiz A, et al. Spanish guidelines for the management of autosomal dominant polycystic kidney disease. Nephrol Dial Transplant. 2014;29 Suppl 4:iv95-105.

3. Chebib FT, Torres VE. Autosomal Dominant Polycystic Kidney Disease: Core Curriculum 2016. Am J Kidney Dis. 2016;67(5):792810

4. Alves EF, Tsuneto LT, Pelloso SM, Torres PR, Otto GL, Silva AA, et al. Autosomal dominant polycystic kidney disease in hemodialysis patients in Southern Brazil. J Bras Nefrol. 2014;36(1):18-25.

5. Herrera-Añazco P, Benites-Zapata V, Hernandez AV, MezonesHolguin E, Silveira-Chau M. Mortality in patients with chronic kidney disease undergoing hemodialysis in a public hospital of Peru. J Bras Nefrol. 2015;37(2):192-7.

\section{Ahora puede enviar sus artículos para Acta Médica Peruana en nuestro Open Journal System:}

www.amp.cmp.org.pe

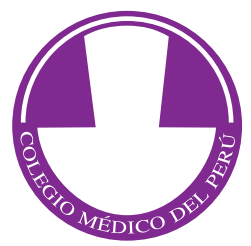

